\title{
Nonlinear Dynamics of a Flexible Portal Frame under Support Excitation
}

\author{
Aline Souza de Paula ${ }^{1}$, José Manoel Balthazar ${ }^{2}$ and Jorge Luis Palacios Felix ${ }^{3}$ \\ ${ }^{1}$ Universidade de Brasilia, Department of Mechanical Engineering, 70.910.900 - Brasilia - DF, Brazil, \\ alinedepaula@unb.br \\ ${ }^{2}$ Universidade Estadual Paulista, Instituto de Geociências e Ciências Exatas, Departamento de Estatística, \\ Matemática Aplicada e Computação-UNESP, CP 178, 13500-230, Rio Claro, SP, Brasil, jmbaltha@rc.unesp.br \\ ${ }^{3}$ Universidade Federal do Pampa, Depto de Matemática 96413-170, Campus Bagé, Bagé - RS, \\ jorge.felix@unipampa.edu.br
}

\begin{abstract}
This paper presents a nonlinear dynamic analysis of a flexible portal frame subjected to support excitation, which is provided by an electro-dynamical shaker. The problem is reduced to a mathematical model of four degrees of freedom and the equations of motion are derived via Lagrangian formulation. The main goal of this study is to investigate the dynamic interactions between a flexible portal frame and a non-ideal support excitation. The numerical analysis shows a complex behavior of the system, which can be observed by phase spaces, Poincare sections and bifurcation diagrams..
\end{abstract}

Keywords: Electro-dynamical shaker, flexible portal frame, nonlinear dynamics.

\section{INTRODUCTION}

In the analyses of vibrating systems, the cases when the external excitation is influenced by system response have been considered as a major challenge in theoretical and practical engineering research. When the excitation is not influenced by system response, it is called an ideal excitation or an ideal energy source. In contrast, when the excitation is influenced by the response of the system, it is known as non-ideal. Different authors studied dynamical system with nonideal excitation (Nayfeh \& Mook, 1979; Balthazar et al., 2003; Dantas \& Balthazar, 2007).

Brasil (1999) performed an analytical study of the nonlinear vibrations of a portal frame excited by two ideal energy sources, consisting of unbalanced rotating machines. This same problem with two non-ideal sources was studied by Balthazar et al. (2004), which focused on the self-synchronization of the energy sources.

Another type of vibrating systems excitation that is analyzed by several authors is the support excitation. $\mathrm{Xu}$ et al. (2007) and De Paula et al. (2011) performed numerical and experimental dynamical analysis of a parametrically excited pendulum. Jiang et al. (2006) studied a tuning fork vibratory micromachined gyroscope subjected to base excitation.

In this paper it is carried out a dynamical analysis of a flexible portal frame under base excitation, which is provided by an electro-mechanical shaker, consisting in a non-ideal energy source. The electromechanical device considered in this work consists of an electric system magnetically coupled to a mechanical structure as used by Felix and Balthazar (2008a and 2008b) and Yamapi (2006) in others vibrating problems.

\section{MATHEMATICAL MODEL}

The proposed system consists of a flexible portal frame (FPF) with base excitation provided by an electro-dynamical shaker, as shown in the schematic picture presented in Figure 1, at deflected position. The system consists of two columns connected by a beam at upper end and clamped in a base at lower end. The base is a platform of mass $m_{0}$ and stiffness $k_{p}$, with vertical displacement denoted by $S$. Each column has height $h$, flexural rigidity of $E I$ and carries a lumped mass $m$ at the upper end. The horizontal beam has length $l$, carries a central mass $M$ and is pinned to the columns at both ends.

The three concentrated masses of the flexible portal frame absolute coordinates can be written as: 


$$
\begin{aligned}
& x_{1}=z_{1}, \quad x_{2}=\frac{l}{2}+z_{2}, \quad x_{3}=l+z_{3}, \quad y_{1}=h-v_{1}+S \\
& y_{2}=h+S-\frac{v_{1}+v_{3}}{2}-v_{2}, \quad y_{3}=h+S-v_{3}
\end{aligned}
$$

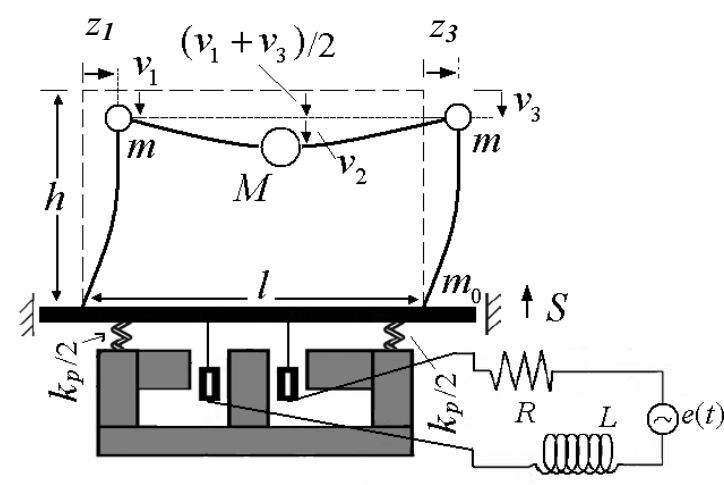

FIGURE 1. Flexible portal frame and electro-dynamical shaker physical model.

The linear stiffness of the columns and the beam can be evaluated by a Raleigh-Ritz procedure from cubic trial functions. Moreover, geometric nonlinearities are introduced by considering the shortening due to columns and beams bending under inextensibility hypothesis as presented by Brasil (1999) and shown in Eq. (2).

$$
\begin{aligned}
& v_{1}=\frac{1}{2} A z_{1}^{2}, v_{3}=\frac{1}{2} A z_{3}^{2}, z_{2}=z_{1}-\frac{1}{4} B v_{2}^{2}, \\
& z_{3}=z_{1}-\frac{1}{2} B z_{2}^{2}
\end{aligned}
$$

where the following constants are also obtained from cubic trial functions:

$$
A=\frac{6}{5 h}, B=\frac{24}{5 l}
$$

Using algebraic relations presented in Eq. (1) and Eq. (2), the modeling of the flexible portal frame system may be reduced to two unknown variables: $z_{1}$ (horizontal displacement) and $v_{2}$ (vertical displacement).

The coupling between the flexible portal frame and the electro-dynamical shaker is then obtained through the electromagnetic force as presented in Eq. (4), caused by a permanent magnet.

$$
F_{e m}=K \dot{q}
$$

where $K=l_{c} B_{c}$, and $l_{c}$ and $B_{c}$ are, respectively, length of the conductor and magnetic flied. As a result, it produces a Laplace force in the mechanical part and the Lenz electromotive voltage in the electrical part. The electrical part of the system consists of a resistor $(R)$, an inductor $(L)$, a capacitor $(C)$ and a sinusoidal voltage source $\left(e(t)=e_{0} \cos (\omega t)\right)$ connected in series. In the present model, the capacitor voltage is a nonlinear function of the instantaneous electrical charge $q$ as presented in Eq. (5).

$$
V_{c}=\frac{1}{C_{0}} q+d_{3} q^{3}
$$

where $C_{0}$ is the linear part of $C$ and $d_{3}$ is the nonlinear coefficient, both depending on the type of the capacitor in use.

The system equations of motion can then be obtained by using the Lagrangian equations and are presented in Eq. (6) in dimensionless form. The portal frame governing equations without excitation are similar to those obtained by Balthazar et al. (2004) and the coupling with the electro-dynamical shaker is obtained according to Felix et al. (2008).

$$
\begin{gathered}
X^{\prime \prime}+\mu_{1} X^{\prime}+X=\alpha_{1}\left(Y^{\prime \prime} Y+Y^{\prime 2}\right)+ \\
+\frac{\alpha_{1}}{2} Y^{2}+\frac{\alpha_{1}}{2} U^{\prime \prime} X-\delta_{1} X Y^{\prime \prime} \\
Y^{\prime \prime}+\mu_{2} Y^{\prime}+w_{2}^{2} Y=\delta_{2} X^{\prime \prime} Y+\gamma_{2} X Y+ \\
-g_{2}-\alpha_{2}\left(X^{\prime 2}+X X^{\prime \prime}\right)+\delta_{3} U^{\prime \prime} \\
U^{\prime \prime}+\mu_{3} U^{\prime}+w_{3}^{2} U-\beta_{1} V^{\prime}=\alpha_{3}\left(X^{\prime 2}+X X^{\prime \prime}\right)+\delta_{4} Y^{\prime \prime}-g_{1} \\
V^{\prime \prime}+\mu_{4} V^{\prime}+w_{4}^{2} V+\lambda_{3} V^{3}+\beta_{2} U^{\prime}=E_{0} \cos \Omega \tau
\end{gathered}
$$

where $\tau=\omega_{1} t, X=\frac{z_{1}}{h}, Y=\frac{v_{1}}{l}, U=\frac{S}{l}, V=\frac{q}{q_{0}}$, $\omega_{1}=\sqrt{\frac{2 k_{c}}{M+2 m}-A g}, \quad \omega_{2}=\sqrt{\frac{k_{b}}{M+m}}$, $w_{2}=\frac{\omega_{2}}{\omega_{1}}, \omega_{3}=\sqrt{\frac{k_{p}}{M+2 m+m_{0}}}, \quad w_{3}=\frac{\omega_{3}}{\omega_{1}}, \quad w_{4}=\frac{\omega_{e}}{\omega_{1}}$, $\Omega=\frac{\omega}{\omega_{1}}, \quad \alpha_{1}=\frac{12 l}{5 h}, \quad \delta_{1}=\frac{\alpha_{1} M}{2(M+2 m)}$, $\mu_{1}=\frac{c_{1}}{(M+2 m) \omega_{1}}, \quad \delta_{2}=w_{2}^{2} \gamma_{2}, \quad \gamma_{2}=\frac{12 h(M+2 m)}{5 l(M+m)}$, $\mu_{2}=\frac{c_{2}}{(M+m) \omega_{1}}, \quad g_{1}=\frac{g}{\omega_{1}^{2} l}, \quad g_{2}=\delta_{3} g_{1}, \quad \alpha_{2}=\frac{6 h \delta_{3}}{5 l}$, 
$\delta_{3}=\frac{M}{M+m}, \quad \mu_{3}=\frac{c_{3}}{M_{t} \omega_{1}}, \quad \beta_{1}=\frac{\mathrm{K} q_{0}}{M_{t} \omega_{1} l}$,

$\alpha_{3}=\frac{6 h(M+2 m)}{5 l M_{t}}, \delta_{4}=\frac{M}{M_{t}}, \mu_{4}=\frac{R}{L \omega_{1}}, \lambda_{3}=\frac{d_{3} q_{0}^{2}}{L \omega_{1}^{2}}$,

$\beta_{2}=\frac{\mathrm{K} l}{L \omega_{1} q_{0}}, \quad E_{0}=\frac{e_{0}}{L \omega_{1}^{2} q_{0}}, \quad \Omega=\frac{\omega}{\omega_{1}} \quad$ and

$M_{t}=M+2 m+m_{0}$.

\section{NUMERICAL RESULTS}

The dynamical analysis of the proposed system is carried out numerically by using the forth order Runge-Kutta method and appropriate tools for nonlinear dynamics investigation. An important characteristic associated with dynamical response of nonlinear system near resonance is the jump phenomena (Bernardini \& Rega, 2005; Savi et al., 2011). Dynamical jumps result in abrupt changes in system behavior and its analysis is essential for a proper design of nonlinear systems. Besides abrupt changes in system response, these jumps lead to system instability due to the coexistence of periodic attractors. These jumps are related to the Sommerfeld effect (Sommerfeld, 1902; Balthazar et al., 2003).
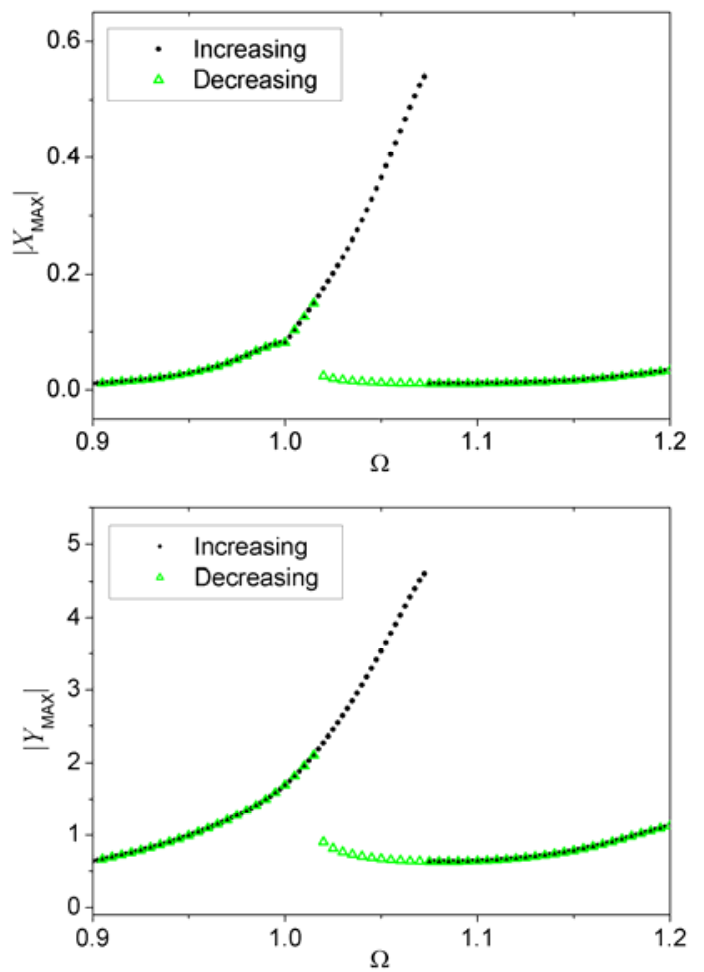

FIGURE 2. Maximum displacement by increasing and decreasing the forcing frequencies.

In this context, Figure 2 shows the maximum $X$ displacement and $Y$-displacement with $E_{0}=0.15$ by increasing and by decreasing the forcing frequency and considering the following parameters: $\mu_{1}=0.05$, $\mu_{2}=0.05, \quad \mu_{3}=0.01, \quad \mu_{4}=0.01, \quad \delta_{1}=0.05, \quad \delta_{2}=0.5$, $\delta_{3}=0.8, \quad \delta_{4}=0.2, \quad w_{2}=0.5, \quad w_{3}=1, \quad w_{4}=1, \quad \alpha_{1}=0.05$, $\alpha_{2}=0.1, \quad \alpha_{3}=0.4, \quad g_{1}=0.001, \quad g_{2}=0.0008, \quad \beta_{1}=0.4$, $\beta_{2}=0.2, \lambda_{3}=0.95, \gamma_{2}=0.05$.

From Figure 2 it can be observed the presence of dynamical jumps and also different behavior of the system when increasing and decreasing the frequency. This different ways cause instability, since the system can present two different stable orbits at the same parameters depending on the initial condition.

Figure 3 presents phase space of the coexistent orbits observed in Figure 2 at $\Omega=1.07$. The black curve is related to system behaviour obtained when increasing the forcing frequency, while the green curve corresponds to system response obtained when decreasing the frequency.
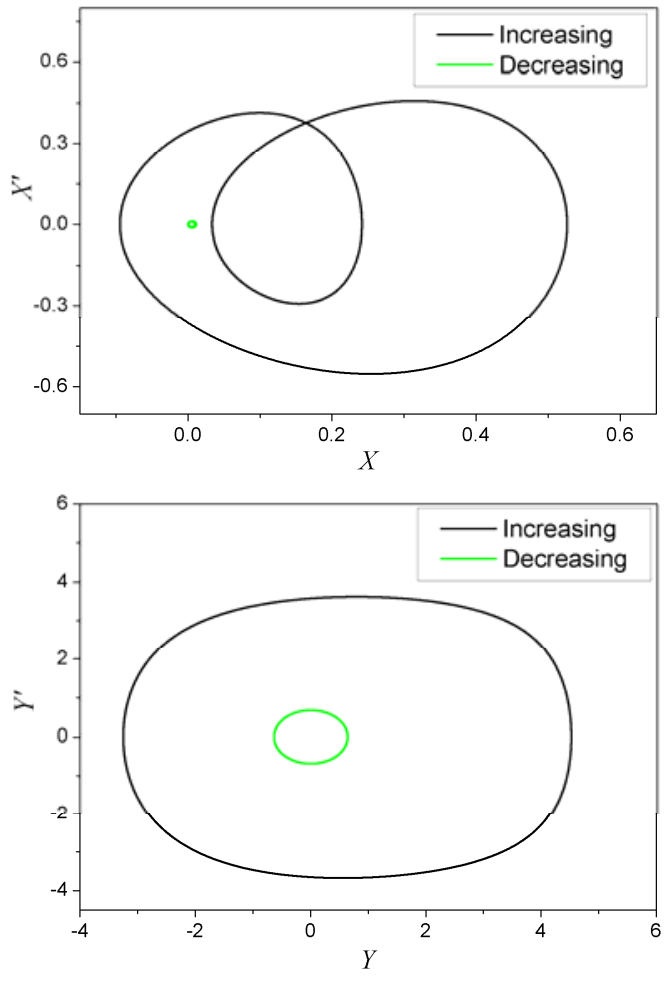

FIGURE 3. Phase space of the coexistent orbits with $E_{0}=0.15$ and $\Omega=1.07$. 
It can be observed a significant difference between the two stable orbits that coexists at the same system and forcing parameters. The change from one orbit to the other can occurs with slight external perturbations. These abrupt changes are dangerous to the dynamical system.

Figure 4 shows electrical charge and portal frame vertical displacement in time so that the energy transfer between the shaker and the portal frame in steady state can be observed. The left figure shows the behavior obtained when increasing the forcing frequency, while the right figure presents the response when decreasing the frequency. In both periodic motions there is a phase angle of $\pi / 2$ between the two variables, where energy transfer from the shaker to the flexile portal frame is verified. It is important to highlight that the portal frame horizontal displacement and the base motion are in the same phase as the presented $y$-displacement.
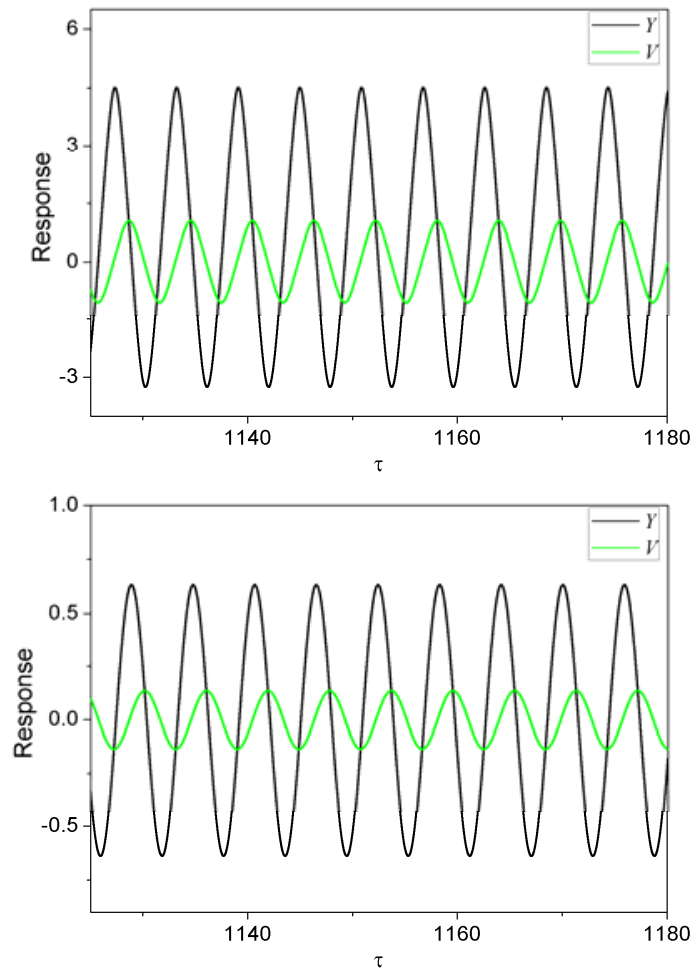

FIGURE 4. Energy transfer between shaker and portal frame with $E_{0}=0.15$ and $\Omega=1.07$. Left: response when increasing the forcing frequency; Right: response when decreasing the forcing frequency.

The dynamical jumps obtained in Figure 2 can also be observed in the bifurcation diagram presented in Figure 5. The bifurcation is constructed by assuming a quasi-static stroboscopically increase and decrease of the forcing frequency, $\Omega$, with $E_{0}=0.15$. Moreover, the first 1000 periods are neglected in order to reach the steady state response.
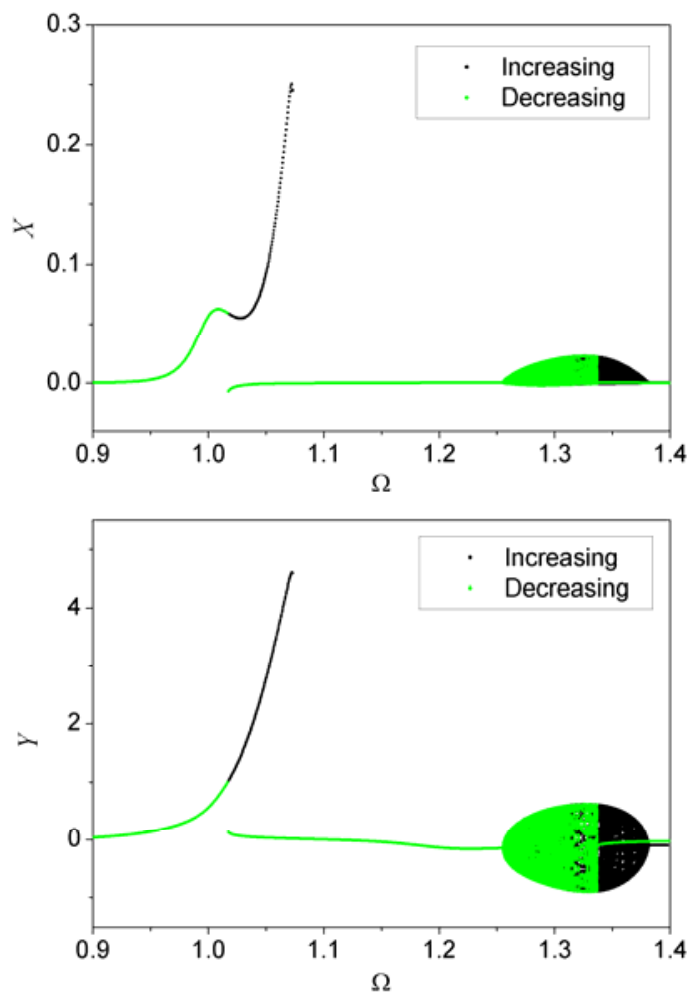

FIGURE 5. Bifurcation diagram constructed by increasing and decreasing the forcing frequencies.

Besides dynamical jumps, the bifurcation diagram indicates a region related to quasi-periodic behavior as well as a region of coexistence of quasi-periodic and periodic attractors. Figure 6 presents these two attractors in phase space with $\Omega=1.35$. The quasiperiodic response is obtained when increasing the forcing frequency, represented by the black curve, while the periodic response is obtained when decreasing the frequency and is represented by the green curve. 

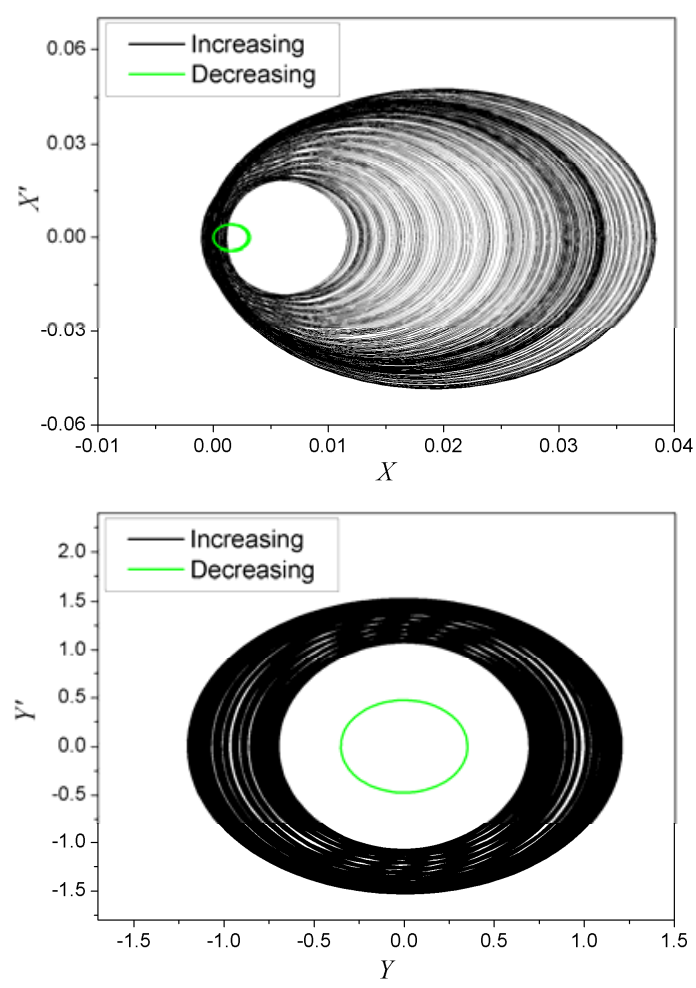

FIGURE 6. Coexistent attractors with $\mathrm{E}_{0}=0.15$ and $\Omega=1.35$.

Figure 7 presents phase space and Poincare section at $x-x^{\prime}$ and $y-y$ ' planes related to periodic orbit presented in Figure 6 . While Figure 8 presents the same results for the quasi-periodic motion.

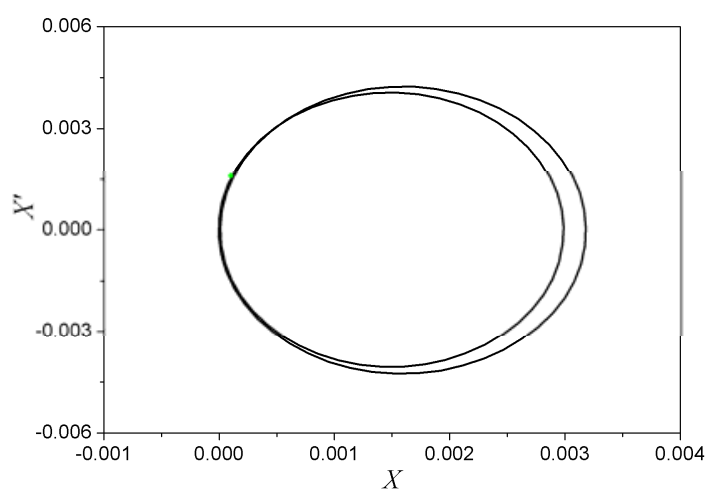

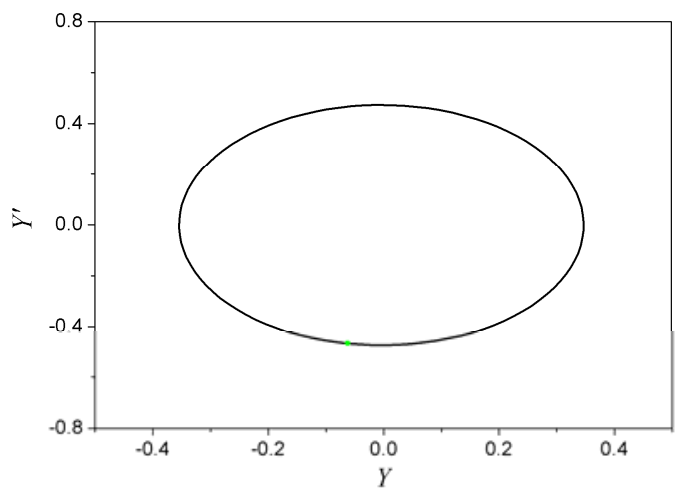

FIGURE 7. Phase space and Poincare with $E_{0}=0.15$ and $\Omega=1.35$. Behavior obtained when decreasing the forcing frequency.
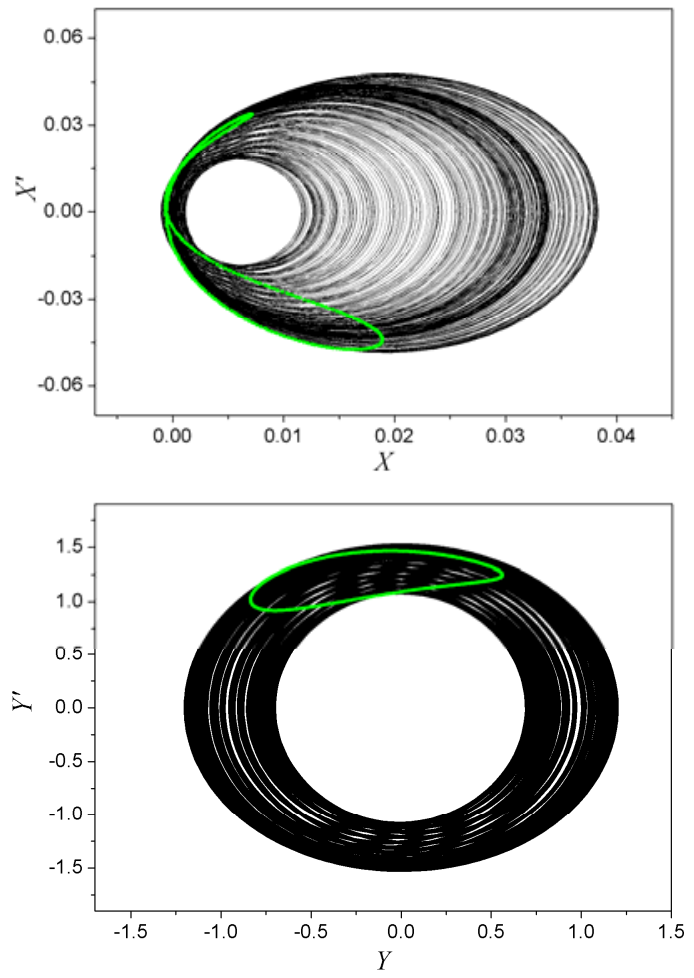

FIGURE 8. Phase space and Poincare with $E_{0}=0.15$ and $\Omega=1.35$. Behavior obtained when increasing the forcing frequency.

Similarly to Figure 4, Figure 9 shows electrical charge and portal frame vertical displacement in time. The left figure shows the behavior obtained when increasing the forcing frequency, while the right figure presents the response when decreasing the frequency. In the case of the periodic orbit (Figure 9 right), the behavior is similar to the ones obtained in Figure 4. In the case of quasi-periodic motion (Figure 9 left), 
however, the energy transfer between shaker and flexible portal frame is more significant, when the shaker electrical charge is increasing, the vertical displacement is decreasing and vice-versa.
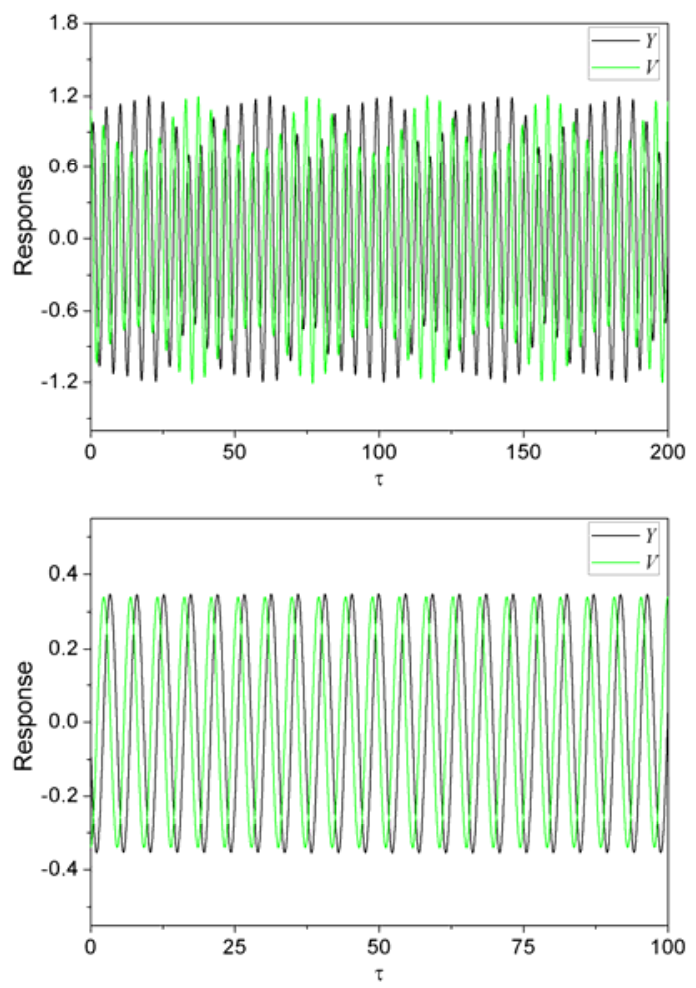

FIGURE 9. Energy transfer between shaker and portal frame with $E_{0}=0.15$ and $\Omega=1.35$. Left: Quasi-periodic behavior; right: periodic behavior.

\section{CONCLUSIONS}

In this work a flexible portal frame parametrically excited by an electro-dynamical shaker is considered. The dynamical analysis of the system is carried out numerically considering different forcing frequencies, mainly near the resonance region. The occurrence of dynamical jumps is verified, which is an important characteristic of nonlinear dynamical systems. Basically, dynamical jumps are associated with nonlinear resonant response, causing abrupt changes in system behavior and introducing unstable regions on system response. The unstable region is caused by the presence of coexisting periodic attractors, as presented in the results. Besides the dynamical jumps, an important point associated with the proposed system is the energy transfer between the shaker and the FPF. This energy transfer is observed when system behaves periodically but it is more significant in quasi-periodic response. In the obtained periodic behavior it is observed energy transfer from shaker to FPF. In the case of quasi-periodic behavior it is verified energy transfer from shaker to FPF and also from FPF to shaker.

\section{ACKNOWLEDGMENTS}

The authors would like to thank DPP-UnB, the Brazilian Research Agencies $\mathrm{CNPq}$ and through the INCT-EIE (National Institute of Science and Technology - Smart Structures in Engineering) the CNPq and FAPEMIG for their support.

\section{REFERENCES}

1. Balthazar, J.M., Mook, D.T., Weber H.I., Brasil, R.M.L.R.F., Fenili, A., Belato, D. \& Felix, J.L.P., 2003, "An Overview on Non-ideal Vibrations", Meccanica, v.38, pp.613-621.

2. Balthazar, J.M., Felix, J.L.P. \& Brasil, R.M.L.R.F., 2004, "Short Comments on Self-Synchronization of the Non-Ideal Sources Supported by a Felxible Portal Frame Structure", Journal of Vibration and Control, v.10, pp. 1739-1748.

3. Bernardini, D. \& Rega, G., 2005, "Thermomechanical modelling, nonlinear dynamics and chaos in shape memory oscillators", Mathematical and Computer Modelling of Dynamical Systems, v.11, n.3, pp.291-314.

4. Brasil, R.M.L.R.F., 1999, "Multiple Scales Analysis of Nonlinear Oscillations of a Portal Frame Fondations for Several Machines", Journal of the Brazilian Society of Mechanical Sciences, v. 21 (4), pp. 641-654.

5. Dantas, M.J. H. \& Balthazar, J. M., 2007, "On the existence and stability of periodic orbits in non-ideal problems: general results", ZAMM- Journal of Applied Mathematics and Mechanics, v.58, pp.940-956.

6. De Paula, A. S., Savi, M. A., Wiercigroch, M. \& Pavlovskaia, E., 2011, "Bifurcation Control of a Parametric Pendulum", International Journal of Bifurcation and Chaos in Applied Sciences and Engineering, Accepted.

7. Felix, J.L.P., Balthazar, J.M., 2008a, "Comments on a Nonlinear and Nonideal Electromechanical Damping Vibration Absorber, Sommerfeld Effect and Energy Transfer", Nonlinear Dynamics, Doi: 10.2007/s11071008-9340-8.

8. Felix, J.L.P., Balthazar, J.M., 2008b, "On a non-ideal and nonlinear tuning fork micro-gyroscope dynamics", In: Twelfth Conference on Nonlinear Vibrations, Dynamics, and Multibody Systems, June 1-5, 2008, VT, Blacksburg, VA, USA.

9. Jiang, T., Wang, A. \& Liu, G., 2006, "Detection capacitance analysis method for tuning fork micromachined gyroscope based on elastic body model", Sensors and Actuators A, v.128, pp.52-59.

10. Nayfeh A. H. \& Mook D. T., 1979, Nonlinear Oscillations, Wiley \& Sons.

11. Savi, M. A.; De Paula, A. S. \& Lagoudas, D., 2011, "Numerical Investigation of an Adaptive Vibration 
Absorber Using Shape Memory Alloys", Journal of Intelligent Material Systems and Structures, v. 22, p. 6780.

12. Sommerfeld, A., 1902, "Beiträge zum dynamischen ausbau der festigkeitslehe", Physikal Zeitschr, v.3, pp.266 and 286.

13. Xu, X., Pavlovskaia, E., Wiercigroch, M., Romeo, F. \& Lenci, S., 2007, "Dynamic interactions between parametric pendulum and electro-dynamical shaker", Z Angew. Math. Mech., v.87, n.2, pp.172 - 186.

14. Yamapi, R., 2006, "Dynamics of an Electromechanical Damping Device with Magnetic Coupling", Communication in Nonlinear Science and Numerical Simulation, v. 11, pp. 907-921. 
Copyright of AIP Conference Proceedings is the property of American Institute of Physics and its content may not be copied or emailed to multiple sites or posted to a listserv without the copyright holder's express written permission. However, users may print, download, or email articles for individual use. 